\title{
DISCURSO ACADÉMICO DE LANZAMIENTO DE LAREVISTA CHILENA DE DERECHO Y CIENCIA POLÍTICA
}

\author{
ACADEMIC SPEECH ON THE LAUNCH OF THE CHILEAN REVIEW OF LAW AND \\ POLITICAL SCIENCE
}

Patricio Ignacio Carvajal ${ }^{1}$

\begin{abstract}
Señoras y Señores,
\end{abstract}
Me complace enormemente la invitación que se me ha cursado para dirigir algunas palabras en el lanzamiento de esta Revista Chilena de Derecho y Ciencia Política . Me honra el hecho de que me hayan tenido Uds. en consideración para este acto académico y, por supuesto, también que me hayan incorporado al elenco de especialistas de su Comité Científico Externo. Por ello, comienzo por expresar mi sincero agradecimiento.

La revolución científica del siglo XVII introdujo cambios notables en lo que hoy Ilamamos metodología del derecho. Por ejemplo, así veía Pufendorf (1632-1694) las operaciones intelectuales involucradas en la construcción jurídica, en su De Jure naturae et gentium $1.2 \S 2^{2}$ :

Demonstrare igitur nobis est, rerum propositarum certitudinem necessariam et certis principiis tamquam suis causis indubitato cognoscendam syllogistice deducere.
En consecuencia, para nosotros demostrar es deducir silogísticamente una certidumbre necesaria de las cosas propuestas y por principios ciertos, vale decir, por sus causas indubitadas de conocimiento.

Podemos encontrar esto mismo sintetizado en Thomasius (1655-1728), en sus Fundamenta juris naturae et gentium $1.6 . \S 1^{3}$ :

1 Abogado. Profesor de Derecho Romano, Pontificia Universidad Católica de Chile. Miembro Comité Científico Internacional Revista Chilena de Derecho y Ciencia Política, Santiago, Chile.

2 Pufendorf, Samuel, De jure naturae et gentium libri octo, Londini Sacanorum, 1672, p. 21. El libro I lleva por título "De origine et varietate entium moralium " (Del origen y variedad de los entes morales); el capítulo II "De certitudine disciplinarum, quae circa moralia versantur " (De las certeza de las disciplinas que versan sobre la moral); y el § 2. "Quid sit demonstratio " (Qué sea la demostración).

3 Cfr. Thomasius, Christian, Fundamenta iuris naturae et gentium ex sensu communi deducta, in quibus

doi: 10.7770/RCHDYCP-V3N1-ART348 
Per principium hic... intelligitur... propositio prima et generalissima iuris naturae unde reliqua omnia deduci possunt.
Por principio aquí... se entiende... la proposición primera y generalísima de derecho de la naturaleza, de donde pueden deducirse todas [sc. las demás proposiciones].

El paradigma de las ciencias naturales, que condujo a esta búsqueda de los principios del derecho con un carácter universal (dotados de la debida precisión y demostrabilidad), también cambió nuestro mundo editorial. De hecho, fue este paradigma lo que trajo de la mano la emersión de revistas, tales como Acta eruditorum, Journal des savants o Transactions of the Royal Society. Aquí se encauzó una costumbre previa de los intelectuales: intercambiar cartas para tratar temas precisos y particulares; lo cual dio lugar a que los artículos de las revistas recibieran el nombre (todavía presente) de "comunicaciones". Comunicar es, en efecto, el principal papel de una revista científica, y, por cierto, de una revista jurídica ${ }^{4}$.

El movimiento intelectual que impulsó el desarrollo de este género editorial, se dejó sentir prontamente en el Chile republicano. Si dejamos de lado las publicaciones (muchas de ellas célebres) que solían hacerse a través de columnas de prensa, y la fugaz existencia de la primera revista chilena de pedagogía, Redactor de la Educación (1825-1826) , encontramos un primer hito en el año 1839. Al interior del Instituto Nacional se acordó realizar reuniones periódicas para presentar los avances científicos y literarios. Asimismo, don Vicente Fidel López inició la Revista de Valparaíso, y don Juan García del Río, El Museo de Ambas Américas. Ya en 1842 los profesores del propio Instituto, presididos por don José Victorino Lastarria, fundaron una sociedad literaria que dio vida a "El Semanario de Santiago", en el cual colaboraron don Antonio García Reyes, don Manuel A. Tocornal, don Salvador San Fuentes y don José Joaquín Vallejos (Jotabeche) ${ }^{6}$.

Pero la primera revista propiamente jurídica de nuestro país (es decir, nuestra primera revista científica), fue la Gaceta de los Tribunales y de la Instrucción Pública. Periódico Ministerial de la República de Chile (1841). Su encargado de redacción

ubique secernuntur principia honesti, justi ac decori, cum adjuncta emendatione ad ista fundamenta institutionum jurisprudentiae divinae [Fundamentos de derecho de la naturaleza y de gentes deducidos desde el sentido común, en los cuales en todas partes se distinguen los principios de lo honesto, de lo justo y de lo decoroso, con enmienda adjunta a estos fundamentos de las instituciones de jurisprudencia divina], viduae Christophori Salfeldii, Halae \& Lipsiae, 1718, p. 167.

4 Vid., en este sentido y aportando estos antecedentes, Guzmán Brito, Alejandro, La Revista Chilena de Derecho y su papel entre las revistas jurídicas de Chile /en/ Revista Chilena de Derecho, № 26,1999, p. 263.

5 Vid. Campbell, Margaret, Education in Chile, 1810-1842 /in/ Journal of Inter-American Studies, 1959, p. 358.

6 Labarca, Amanda, Historia de la enseñanza en Chile, Imprenta Universitaria, Santiago, 1939, p. 102. 
(hoy diríamos, Director), fue don José Gabriel Palma, con la colaboración, entre otros, de don Antonio García Reyes, don José Antonio Álvarez y don Antonio Varas ${ }^{7}$. Dicha revista, de índole estatal y no universitaria, nació junto con la estabilización y pacificación nacional que supuso el gobierno de don Manuel Bulnes Prieto. Había quedado atrás la guerra contra la Confederación Perú-boliviana (1837-1839); al igual que las convulsiones internas, cuyo episodio más simbólico fue el asesinato de don Diego Portales (en 1837). A cambio, comenzaba una época de progreso espiritual de la nación, el cual tiene como hito más significativo la fundación de la Universidad de Chile (1842).

La edición número 1 de la Gaceta de los Tribunales, del 6 de noviembre de 1841, se abre en la primera columna de su primera página con un "Prospecto", en el cual el redactor fija los objetivos de la publicación. Quisiera rescatar los siguientes pasajes:

"Cuando una nacion está libre de enemigos esteriores que combatir y en su interior no hai divisiones de partidos que turben la tranquilidad, el Gobierno debe pensar en mejorar sus instituciones, reformar los abusos y formar buenas costumbres... En este periódico se van a ver por ahora con prontitud las sentencias de los tribunales... Noticias estadísticas de los delincuentes, de los delitos, de la materia de los pleitos, y otras igualmente útiles se imprimirán tambien con la exactitud y oportunidad que puedan proporcionar las oficinas y ajentes del Gobierno de una nacion jóven que apénas entra a la pubertad. Documentos de la historia de nuestro derecho, piezas legales inéditas o no bien conocidas, y todo lo que conduzca a la ilustracion de las personas que quieran estudiar la ciencia del foro, tendrán cabida segun su mayor utilidad. Los editores no vamos a constituirnos censores... Las columnas de este papel estarán a disposicion del que quiera tratar una cuestion importante de jurisprudencia o lejislacion... El triunfo de la verdad y el respeto a las autoridades es nuestra divisa: estamos convencidos de que se puede censurar los errores, indicar las faltas, y respetar al mismo tiempo las personas que sean apreciables por otros títulos" ${ }^{\prime \prime}$.

Así quedaban sentadas las bases de esta revista en su ámbito jurídico. Pero, tal como lo anunciaba su título, también se pretendía abordar materias relativas a la instrucción pública. Sobre ello, el "Prospecto" citado remata diciendo:

"La instruccion de la juventud ha sido nuestro ídolo, ella es la esperanza de todos los que han tenido la esperanza de contribuir al establecimiento del nuevo órden y de la independencia y libertad de que gozamos"

7 Vid. Guzmán Páez, Rodrigo, Índice de la Revista "Gaceta de los Tribunales" 1841-1860 /en/ Revista de Estudios Histórico-Jurídicos , 1983, p. 169.

8 Gaceta de los Tribunales, 1841, p.1.

9 Ibid. 
Los contenidos previstos: la jurisprudencia, las noticas jurídicas y la doctrina histórica y actual, pueden considerarse valiosos y necesarios hasta hoy. Pongo de relieve que la Gaceta de los Tribunales tenía por principio "el triunfo de la verdad y el respeto de las autoridades", con el fin de coadyuvar al Gobierno para "mejorar sus instituciones, reformar los abusos y formar buenas costumbres". El redactor tenía por meta beneficiar a los partícipes del foro y a los responsables de los destinos de la nación; pero ello, no menos que a los estudiantes; respecto de quienes -merced al auspicioso ambiente de paz alcanzado-, confesaba con entusiasmo que su formación intelectual "es la esperanza de todos los que han tenido la esperanza de contribuir al establecimiento del nuevo órden y de la independencia y libertad de que gozamos".

Como suele ocurrir en la pesquisa histórica, encontramos aquí indudables aciertos que Ilaman a la reflexión actual. Desde luego, Ilama la atención que todo lo señalado hoy podría coordinarse perfectamente con la Constitución Apostólica sobre la Universidades Católicas, Ex Corde Ecclesiae. La introducción de este documento, como se sabe, reza así:

"Nacida del corazón de la Iglesia, la Universidad Católica se inserta en el curso de la tradición que remonta al origen mismo de la Universidad como institución, y se ha revelado siempre como un centro incomparable de creatividad y de irradiación del saber para el bien de la humanidad. Por su vocación la Universitas magistrorum et scholarium se consagra a la investigación, a la enseñanza y a la formación de los estudiantes, libremente reunidos con sus maestros animados todos por el mismo amor del saber..."10. Y algo más adelante añade: "En este contexto, las Universidades Católicas están llamadas a una continua renovación, tanto por el hecho de ser universidad, como por el hecho de ser católica... Tal renovación exige la clara conciencia de que, por su carácter católico, la Universidad goza de una mayor capacidad para la búsqueda desinteresada de la verdad; búsqueda, pues, que no está subordinada ni condicionada por intereses particulares de ningún género"11.

Retornando a la Gaceta de los Tribunales, debemos añadir que a pesar de haber perfilado, en su día, tan acertadamente sus objetivos, estos, a poco andar, parecen haber resultado demasiado ambiciosos. Lamentablemente, padeció un relativamente rápido decaimiento científico. De modo que a partir de 1860 se limitó únicamente a reproducir fallos ${ }^{12}$. Así pervivió hasta 1950, momento en el que, a instancias de don Arturo Alessandri Rodríguez ${ }^{13}$, se fusionó con otra de signo más científico y de géne-

10 Ex corde Ecclesiae, Introducción, 1.

11 Id. 7.2 .

12 Vid. Guzmán Páez, Rodrigo, obr. cit., p. 169.

13 Abogado Ilustre. Don Arturo Alessandri Rodríguez /en/ Revista del Abogado, N 4, 1995, p. 40.

182 Revista Chilena de Derecho y Ciencia Política - ISSN Impreso: 0718-9389 / ISSN on line: 0719-2150 
sis universitaria, que había nacido en 1903-1904 (en medio de la promulgación del Código de Procedimiento Civil y de la reforma curricular de los estudios de Derecho en la Universidad de Chile): la Revista de Derecho, Jurisprudencia y Ciencias Sociales. Esta, a su turno, debía su existencia al empeño inicial de don Eliodoro Yáñez y don Luis Claro Solar ${ }^{14}$. Como resultado de la confluencia indicada, desde 1951 apareció la Revista de Derecho y Jurisprudencia y Gaceta de los Tribunales; la cual permitió que la Gaceta reasumiera su misión científica original.

En un rápido (y, por tanto, incompleto) recuento de la emersión de las revistas jurídicas, ahora al amparo de las universidades, no se puede dejar de mencionar otra revista universitaria: la Revista de Derecho de la Universidad de Concepción, la cual nació en 1933, pero lamentablemente se descontinuó el año 2008. En realidad, en materia de revistas jurídicas nacionales, yo he manejado al menos un centenar. $Y$ se observa con facilidad que la mayoría de ellas ya no están vigentes: es más, varias han comenzado y terminado con su primer número. Este es un llamado de atención en cuanto a la responsabilidad que pesa sobre los editores.

La revista a cuyo lanzamiento hoy asistimos, ha decidido llamarse Revista Chilena de Derecho y Ciencia Política. Con ello, pasa a engrosar la lista de "revistas chilenas de derecho" ya existentes: la Revista Chilena de Ciencia Penitenciaria y de Derecho Penal (iniciada en 1950); la Revista Chilena de Historia del Derecho (iniciada en 1959); la Revista Chilena de Derecho (iniciada en 1974); la Revista Chilena de Derechos Humanos (iniciada en 1985); y la Revista Chilena de Derecho Privado (iniciada en 2003).

De entre todas estas, creo que, dada la afinidad de tratarse de una revista editada por una universidad católica, además de otros múltiples méritos, cabe destacar hoy la Revista Chilena de Derecho de la Pontificia Universidad Católica de Chile (fundada, como se dijo, en 1974). Ella recogió experiencias anteriores de su Facultad editora: los Anales Jurídico-Sociales (1936-1962) y los Estudios Jurídicos $(1972-1973)^{15}$. Hoy, es la revista universitaria de Derecho más antigua del país; una vez encargada la Revista de Derecho y Jurisprudencia y Gaceta de los Tribunales a la Editorial Jurídica de Chile, y cesada la Revista de Derecho de la Universidad de Concepción. Asimismo, a diferencia de las demás revistas chilenas de derecho, es la única de carácter general. O, más bien, lo era. Pues ahora, esta revista, la Revista Chilena de Derecho y Ciencia Política, nace también con vocación general y hasta multidisciplinar. Cuando se inauguró la Revista Chilena de Derecho de la Pontificia Universidad Católica de Chile, cuyo primer Director fue don Jaime Navarrete Barrue-

14 Alessandri, Arturo, Somarriva, Manuel, Vodanovic, Antonio, Tratado de Derecho Civil. Partes Preliminar y General, I., p.132.

15 Gaete Rojas, Sergio, Editorial /en/ Revista Chilena de Derecho, №1, 1974, p. 5. 
to ${ }^{16}$, el Decano, don Sergio Gaete Rojas, indicó los contenidos que tendría ${ }^{17}$-los cuales no distaban de los de la Gaceta de los Tribunales-. Al cumplirse 25 años de la Revista Chilena de Derecho, fue invitado a impartir una conferencia el querido amigo y colega, Prof. Alejandro Guzmán Brito (a la sazón, desde 1979, fundador y director de la prestigiosa Revista de Estudios Histórico-Jurídicos). Allí destacó, como dos virtudes fundamentales, la continuidad de nuestra publicación y, precisamente, la estabilidad de sus contenidos ${ }^{18}$. Pero, en honor a la verdad, el Decano Gaete en el lanzamiento había propuesto un objetivo que en 1999 se había cumplido solo parcialmente: transformar la revista en "un vehículo de comunicación de la actividad jurídica chilena con la extranjera"19. Hace poco, al cumplirse los 35 años de la revista, su actual directora, y también querida amiga, Profa Ángela Vivanco, me honró con encargarme la conferencia central del acto $^{20}$. En esa ocasión recordé las palabras del recordado e importante Decano Gaete. La situación había cambiado radicalmente en los diez últimos años. Observé, en lo que respecta a la internacionalización de sus autores y sus temáticas, la importancia de su incorporación al consorcio SCIELO, a Scopus y a otros índices internacionales de relevancia. Este avance es un reflejo de los cambios experimentados en las Facultades de Derecho de Chile; y, especialmente, del impulso que hemos recibido los investigadores a través de concursos públicos como FONDECYT y otros propios de cada Casa de Estudios. Por cierto, las revistas han aumentado su calidad en la medida que la investigación se ha desarrollado. Los contenidos ya no dejan intuir que son piezas forenses reutilizadas para su publicación, sino que son obras propiamente científicas: bien fundamentadas en la experiencia nacional y extranjera, histórica y actual; dotadas de la condición básica que ellas deben tener: su carácter desinteresado (lo cual -no puedo dejar de poner de relieve-, también es propugnado por la Constitución Ex corde Ecclesiae). Si señalo aquí todo esto, es porque me parece que, ahora, la Revista Chilena de Derecho y Ciencia Política debe enfrentar estos mismos desafíos.

Ha quedado dicho desde el inicio, que el paradigma científico del siglo XVII sirvió de marco a la creación de las revistas jurídicas. Ahora bien, este paradigma, hoy superado, condujo hacia un cierto encapsulamiento científico. Esto no era bueno. Por ello me alegra enormemente el carácter multidisciplinar de la publicación que hoy presento. No es que se deba negar la independencia de cada ciencia (hacia lo cual,

16 Vid., sobre el elenco de sus directores, secretarios y miembros de comité editorial, Lecaros, Raúl, Homenaje a los editores de la Revista Chilena de Derecho /en/ Revista Chilena de Derecho № 26, 1999, pp. 256-258.

17 Gaete Rojas, Sergio, obr. cit., pp. 5-6.

18 Guzmán Brito, Alejandro, La Revista Chilena de Derecho y su papel entre las revistas jurídicas de Chile, /en/ Revista Chilena de Derecho N²6, 1999, pp. 263-265.

19 Gaete Rojas, Sergio, obr. cit., pp. 5-6.

20 Carvajal, Patricio-Ignacio, Nuevas Pautas Investigativas en la Ciencia Jurídica (Derecho: ley y ciencia, sociedad e historia) /en/ Revista Chilena de Derecho, N³7, 2010, pp. 191-196. 
lamentablemente, propende la interdisciplinariedad cuando es mal entendida), sino que se debe constatar que todo objeto de estudio se puede mirar desde varios puntos de vista; y que esta mirada amplia ciertamente enriquece. Sobre la circunstancia de que "todas las verdades se tocan" versó el discurso del Rector don Andrés Bello en la instalación de la Universidad de Chile $(1843)^{21}$; y en la misma órbita tenemos que situar, hoy, una vez más, nuestra Constitución Apostólica Ex corde Ecclesiae.

Durante el propio siglo XVII, el insigne teólogo-jurista Francisco Suárez (15481617) remarcaba esto. Junto con reconocer los ámbitos propios de la teología, de la moral y del derecho, reclamaba la pertinencia de dar una mirada teológica también a las leyes humanas. En esto no andaba desencaminado, pues conocía bien los límites de su quehacer. Decía críticamente cuál era el pensamiento contrario de su época:

Si enim theologus de legibus agit, quatenus a Deo legislatore derivantur, profecto alienum faciet si ad alios divertat legislatores. Adde quod, cum theologia sit doctrina supernaturalis, ei debet prohiberi descensus ad ea quae hauriuntur a natura et nihil supra illa evehuntur; alioqui naturalis philosophus ultra naturales leges divina etiam consideret; iuris caesarei vel etiam pontificii professores sibi usurpent divinarum legum documenta, quod in promptu est quam sit a scientiarum concordi divisione alienum. ${ }^{1}$
Ciertamente, si el teólogo trata respecto de las leyes, en cuanto se derivan de Dios legislador, hace incursión en lo ajeno si se desvía hacia otros legisladores. Añádase que, como sea la teología una doctrina sobrenatural, a esta debe ser prohibido el descenso a la que parte de la naturaleza y no se eleva en nada sobre aquella; por el contrario, tampoco el filósofo natural, más allá de las leyes naturales, considere la divina; o los profesores cesáreos o también los pontificios usurpen para sí los documentos de las leyes divinas, puesto que es palmario que [esto] sea ajeno de una división concorde de las ciencias.

Hoy, en las ciencias humanas que nos ocupan, no podemos dejar de mirar otras ciencias; las cuales por cierto no suplantan, sino que contribuyen auxiliarmente a la propia. En la Alemania del siglo XIX, el gran Puchta, enseñaba:

Ein ausgebildetes System der Philosophie ist keineswegs als Voraussetzung der Jurisprudenz zu betrachten, und noch weniger kann an den Juristen selbst die Anforderung gestellt werden, daß er Philosoph von Profession sey. Aber er wird ohne philosophische Anschauungen seinem Beruf nicht vollständig entsprechen können ${ }^{2}$
Un sistema de filosofía sofisticado no tiene consistencia para considerarse como requisito de la jurisprudencia, y menos aún puede exigirse al jurista mismo el requisito de que él sea filósofo de profesión. Pero el que no tenga un punto de vista filosófico de su profesión no puede satisfacerla plenamente.

21 Anales de la Universidad de Chile, 1843-1844, pp.16-38. 
Y es que el Derecho no se reduce a un agregado de normas legales. El propio Savigny ya lo había dicho:

Zuletzt ist noch bey dem Code über dasjenige zu sprechen, was in subsidium gelten soll, wo er nicht zureicht. Ueber den Umfang und die Wichtigkeit desselben haben sich die Franzosen nicht getäuscht, sie haben eingesehen, daß eigentlich die allerwenigsten Rechtsfälle unmittelbar durch eine Stelle des Code entschieden werden können, daß also fast überall jenes unbekannte das wahrhaft entscheidende seyn müsse. ${ }^{3}$
Por último, todavía hay que hablar en el Code sobre el qué deba aplicarse in subsidium, donde este no sea suficiente. Sobre la medida y la importancia de esto no se han engañado los franceses, ellos han visto, que realmente son muy pocos los casos de derecho que podrían ser decididos inmediatamente por un pasaje del Code, de manera que casi siempre la verdadera decisión tiene que ser desconocida.

Savigny se refiere a todo aquello que conforma el sistema de conceptos jurídicos. Lo cual, a pesar de constituir una superación de los estrechos márgenes del iusnaturalismo racionalista, todavía era demasiado restringido. Así, Ihering, el otro gran gigante del derecho alemán del siglo XIX, va a indicar los aspectos materiales, la vida misma, como parte de nuestro objeto de estudio:

Kurz die juristischen Grundbegriffe verändern sich im Lauf der Zeit eben so gut, wie die Rechtssätze, und sie müssen es, denn sie sind ja keine blossen logischen Kategorien, sondern die Concentrationsform materialer Rechtssätze, die Rechtssätze aber wechseln mit den Verhältnissen und den Bedürfnissen des Lebens. Und die Unveränderlichkeit der römischen Rechtsbegriffe zu glauben ist eine völlig unreise Vorstellung, die von einem gänzlich unkritischen Studium der Geschichte zeugt ${ }^{4}$.
En síntesis, igualmente el concepto jurídico fundamental cambia en el transcurso del tiempo, como las reglas jurídicas, y esto debe ser, puesto que no son solo categorías lógicas, sino la forma de concentración de reglas jurídicas materiales, las reglas jurídicas cambian empero con las relaciones y las necesidades de la vida. Y la inmutabilidad de los conceptos jurídicos romanos es una noción completamente inmadura, que se manifiesta desde un estudio totalmente acrítico de la historia

El legado fundamental de Ihering (de tanto impacto en Valentín Letelier) ${ }^{22}$, no ha perdido vigencia en ciertos puntos. Y así lo encontramos en el insigne romanista y civilista italiano, del siglo XX, Emilio Betti.

22 Letelier, Valentín, Génesis del derecho y de las instituciones civiles fundamentales, Hume \& Walker (Santiago) / Cabaut y Cía. (Buenos Aires), Imprenta Universitaria, Santiago, 1919, XI + 728 pp. 
Una giurisprudenza che si limitasse all'analisi logica del linguaggio legislativo sottoponendo a critica le espresioni e le formulazioni imprecise che vi si rinvengono, per sostituire ad un discorso inadeguato un discorso imperniato su formole e definizioni rigorose dei concetti in discussione, sarebbe una inconcludente "Begriffsjurisprudenz", la quale fallirebbe al suo fondamentale còmpito ermeneutico proprio perchè, sotto l'influenza di una prevenzione dottrinaria, ignorerebbe i problema di convivenza affrontati dalla legge e i criteri di valutazione che ne governano la soluzione ${ }^{5}$.
Una ciencia jurídica que se limitase al análisis lógico del lenguaje legislativo sometiendo a crítica las expresiones y las formulaciones imprecisas que se encuentren, para sustituir a un discurso inadecuado un discurso centrado en fórmulas y definiciones rigurosas de los conceptos en discusión, sería una inconcluyente "Begriffsjurisprudenz" [jurisprudencia de conceptos], la cual fallaría en su tarea hermenéutica fundamental precisamente porque, bajo la influencia de una prevención doctrinal, ignoraría los problemas de convivencia afrontados por la ley y los criterios de valoración que gobiernan la solución.

En esta ocasión no es pertinente profundizar más sobre estos aspectos de metodología de la ciencia del derecho. Al haberlos puesto de manifiesto, no pretendo otra cosa que acreditar la profunda y sincera alegría intelectual que me provoca la aparición de esta Revista Chilena de Derecho y Ciencia Política. Los frutos de una reunión temática como esta no podrán sino ser motivo de orgullo para sus sostenedores.

Una revista jurídica, como toda la literatura jurídica, debe tener vocación de permanencia, de antigüedad. Esto, porque el derecho no se "registra" en libros, sino que "está" en los libros. Nosotros somos científicos sin laboratorios, nuestro laboratorio es la historia y la lógica, nuestros instrumentos de medición son la prudencia y la sensibilidad social. Por ello, una obra jurídica no pasa de moda, como en otras ciencias. Un estudio de derecho puede llegar a ser útil por más de dos mil años (como de hecho ocurre en el núcleo de nuestro derecho); no solo a través de una vigencia permanente (que también), sino por lapsos de "adormecimiento" y "reflorecimiento" de una institución; sea que renazca en su misma función y características, o en un remozado emplazamiento sistemático. Por ello, los libros de derecho de una biblioteca ¡no se tiran! Ni los criterios de espacio físico ni los índices de uso-consulta ni los criterios de descarte, procedentes de otras ciencias o de simples razones prácticas, podrían negar un hecho básico: los escritos sobre derecho, con el tiempo, pasan a ser el derecho mismo.

Yo deseo que esta Revista Chilena de Derecho y Ciencia Política llegue a ser parte de nuestro derecho. A juzgar por los méritos de sus profesores y por el respaldo de su Universidad, esto muy seguramente llegará a ser así. 


\section{Bibliografía}

Suárez, Francisco, De legibus, I. De Natura Legis, Prooemeio, Corpus Hispanorum de Pace, Consejo Superior de Investigaciones Científicas, Instituto Francisco de Vitoria, Madrid, 1971.

PuCHTA, Georg Friedrich, Cursus der institutionen. Geschichte des Rechts bey dem römischen Volk, mit einer Einleitung in die Rechtswissenschaft, Breitkopf und Härtel, Leipzig, 1856.

Savigny, Friedrich Karl vom, Beruf unsrer Zeit für Gesetzgebung und Rechtswissenschaft, Mohr und Zimmer, Heidelberg, 1814.

IHERING, Rucah, Geist des römischen Rechts, auf den verschiedenen Stufen seiner Entwicklung, III.1, Breitkopf und Härtel, Leipzig, 1888.

Betтı, Emilio, Teoria generale della interpretazione, 2, Dott. A. Giuffrè Editore, Milano, 1955.

188 Revista Chilena de Derecho y Ciencia Política - ISSN Impreso: 0718-9389 / ISSN on line: 0719-2150 